\title{
IRREDUCIBLE NUMERICAL SEMIGROUPS HAVING TOMS DECOMPOSITION
}

\author{
M.A. MORENO, J. NICOLA, AND E. PARDO
}

\begin{abstract}
In this paper we prove that if $S$ is an irreducible numerical semigroup and $S$ is generated by an interval or $S$ has multiplicity 3 or 4, then it enjoys Toms decomposition. We also prove that if a numerical semigroup can be expressed as an expansion of a numerical semigroup generated by an interval, then it is irreducible and has Toms decomposition.
\end{abstract}

\section{INTRODUCTION}

In the last 30 years, $K$-theory provided invariants in order to classify $C^{*}$-algebras. The interest has been also focussed on determining the range of these invariants. In this line, one of the questions was to find a simple $C^{*}$-algebra $A$ whose ordered $K_{0}$-group fails unperforation property.

This question was answered in the affirmative by J. Villadsen [12]. Subsequent refinements, due to Rørdam and Villadsen [9], and Elliott and Villadsen [2], allowed to restrict the $K$ theoretical scope, by constructing a simple $C^{*}$-algebra $A$ such that $\left(K_{0}(A), K_{0}(A)^{+}\right) \cong(\mathbb{Z}, S)$, where $S \subseteq \mathbb{Z}^{+}$is a submonoid such that $\mathbb{Z}^{+} \backslash S$ is a finite set. The natural representation problem is then whether it is possible to find such an algebra for any such monoid $S$. In this direction Toms [11] gives techniques for constructing a simple $C^{*}$-algebra with stable rank one whose ordered $K_{0}$-group is isomorphic to $\mathbb{Z}$ with positive cone

$$
\text { (*) } S=\frac{1}{L}\left(\bigcap_{i=1}^{N}\left\langle q_{i}, m_{i}\right\rangle\right) \cap \mathbb{Z},
$$

where $q_{1}, \ldots, q_{N}$ are prime numbers, $m_{1}, \ldots, m_{N}$ are natural numbers with g.c.d. $\left(q_{i}, m_{i}\right)=1$, and $L \in \mathbb{N}$ with g.c.d. $\left(q_{i}, L\right)=$ g.c.d. $\left(m_{i}, L\right)=1$. The obvious question, posed by Toms, in order to give complete answer to the representation problem (as well as for the structural knowledge of numerical semigroups), is whether any submonoid $S \subset \mathbb{Z}^{+}$with $\mathbb{Z}^{+} \backslash S$ finite should be of this particular form $(*)$.

Toms showed that the answer is affirmative for 2-generated numerical semigroups [10]. In a recent work [5], the authors and $\mathrm{H}$. Thomas proved that the answer to Toms' question is negative, and in fact that there exist infinitely many numerical semigroups which do not

2000 Mathematics Subject Classification. Primary 06F05; Secondary 46L80.

Key words and phrases. Numerical semigroup, Toms decomposition.

The first and third authors were partially supported by the DGI and European Regional Development Fund, jointly, through Project MTM2004-00149, by PAI III grant FQM-298 of the Junta de Andalucía. The third author is also partially supported by the Comissionat per Universitats i Recerca de la Generalitat de Catalunya. The second author is partially supported by an Erasmus-Socrates grant of the European Community. 
entail a Toms decomposition. In spite, the same work shows that the blocks $\frac{1}{L}\left\langle q_{i}, m_{i}\right\rangle \cap \mathbb{Z}$ appearing in the decomposition $(*)$ satisfy interesting regularities (of geometrical nature), so that it is an interesting question to state whether concrete families of numerical semigroups have Toms decomposition.

In this paper, we shows that irreducible numerical semigroups lying in some largely studied classes (e.g. numerical semigroups generated by intervals) has Toms decompositions, and we give explicit expressions of such decompositions.

Let us summarize the contents of this paper. In Section 2 we will establish basic results on numerical semigroups and we will present the connection with Toms' question. In Section 3 , we present a kind of semigroups, the numerical semigroups generated by intervals, we characterize irreducibility for this family, and we prove that these semigroups have Toms decomposition. In the Section 4 we present others families of semigroups having the same decomposition properties.

\section{Numerical SEMigroups AND TOMS DECOMPOSITION}

In this section we provide the necessary definitions and results related to numerical semigroups, to make clear the connection between them and Toms' question.

A numerical semigroup is a subset of $S$ of $\mathbb{Z}^{+}$closed under addition, such that $0 \in S$ and $S$ generates $\mathbb{Z}$ as a group. By definition (see [6]), $\mathbb{Z}^{+} \backslash S$ is a finite set. We refer to the greatest integer not in $S$ as the Frobenius number of $S$ (also called the Conductor of $S$ ) and we denote it by $C(S)$.

We say that a numerical semigroup is irreducible if it can not be expressed as an intersection of two numerical semigroups containing it properly. It is known [8] that $S$ is irreducible if and only if $S$ is maximal in the set of all numerical semigroups with Frobenius number $C(S)$. By [1] and [3], the class of irreducible semigroups with odd (respectively even) Frobenius number is the same as the class of symmetric (respectively pseudo-symmetric) numerical semigroups. Also, every numerical semigroup with two generators is irreducible. The essential point is that every numerical semigroup $S$ admits a decomposition $S=S_{1} \cap S_{2} \cap \ldots \cap S_{n}$ with $S_{i}$ irreducible for all $i$ (see [7]).

We know (see [1] and [6]), that a numerical semigroup $S$ has a unique minimal system of generators $\left\{n_{1}<n_{2}<\ldots<n_{p}\right\}$. We refer to the numbers $n_{1}$ and $p$ as the multiplicity and embedding dimension of $S$ and denote them by $m(S)$ and $\mu(S)$, respectively. Moreover, if $S$ is a irreducible numerical semigroup, $m(S)$ and $\mu(S)$ are linked [8, Proposition 6]. Notice that, if $S$ is a irreducible numerical semigroup and $S=\left\langle n_{1}, n_{2}, \ldots, n_{k}\right\rangle$ is written with the minimal number of generators, then there exists an upper bound for $n_{k}$. Since $n_{k}$ has to be smaller than the Frobenius number of $\left\langle n_{1}, n_{2}\right\rangle$ then $n_{k}<n_{1} n_{2}-n_{1}-n_{2}$. Moreover, by [8, Proposition 6], when the upper bound $k$ is larger than 4 , we have $k \leq n_{1}-1$.

To check the irreducibility of a numerical semigroup with a minimal set of generators of large cardinality is not always easy. We recall a definition that furnishes a helpful device in this context. Let $S$ be a numerical semigroup and $n \in S \backslash\{0\}$. For any $1 \leq i \leq n$, denote by $w(i)$ the smallest element of $S$ congruent with $i-1$ modulo $n$. Notice that this means $w(i)=\min (S \cap(i-1+n \mathbb{Z}))$. We denote by $A p(S, n)=\{0=w(1), \ldots, w(n)\}$ the Apéry set of $n$ in $S$. By [6] we know that $A p(S, n)=\{x \in S: x-n \notin S\}$ and $w(n)=C(S)+n$. 
Definition 2.1. Given a numerical semigroup $S$, we say that $S$ has a Toms decomposition provided that there exist $L, q_{i}, m_{i} \in \mathbb{Z}^{+}$pairwise coprime, with $q_{i}$ prime for $i \in\{1, \ldots, N\}$, such that

$$
S=\frac{1}{L}\left(\bigcap_{i=1}^{N}\left\langle q_{i}, m_{i}\right\rangle\right) \cap \mathbb{Z} .
$$

Next result states that a Toms decomposition is, in fact, an intersection of building blocks of the form $\frac{1}{L}\left(\left\langle q_{i}, m_{i}\right\rangle\right)$ sharing a common $L$.

Lemma 2.2. With the notation of Definition 2.1, we have

$$
\frac{1}{L}\left(\bigcap_{i=1}^{N}\left\langle q_{i}, m_{i}\right\rangle\right) \cap \mathbb{Z}=\bigcap_{i=1}^{N}\left(\frac{1}{L}\left\langle q_{i}, m_{i}\right\rangle \cap \mathbb{Z}\right) .
$$

In [10], Toms proves any numerical semigroup with two generators has a Toms decomposition. Concretely we have the following result

Lemma 2.3. ([10, Lemma 3.3.1]) Let $m$ and $k$ be coprime positive integers. Then there exists a prime $q$ and a positive integer $L$ coprime to both $m$ and $q$ such that

$$
\frac{1}{L}\langle m, q\rangle \cap \mathbb{Z}=\langle m, k\rangle .
$$

The idea of the proof is the following: Consider the sequence of integers $a_{n}=-m+n k$, and let $n_{0}$ be a positive integer such that $a_{n_{0}}$ is positive. Then, $a_{n_{0}}$ is coprime to $k$, and the sequence $\left\{a_{n}: a_{n}>0\right\}$ is arithmetic; in particular, this sequence contains infinitely many primes. Choose a positive integer $L$ such that $a_{L}$ is both prime and greater than $m k-m-k$, and set $q=a_{L}$. Then, the result holds.

Thus, Toms' question has to be proved for numerical semigroups with more than two generators. Moreover, intertwining Toms' argument [10] with the following result, we are allowed, given $S$ a numerical semigroup, to look for a triple of positive pairwise coprime integers $q, m, L$ such that $S=\frac{1}{L}\langle m, q\rangle \cap \mathbb{Z}$, without paying attention to $q$ being prime or not.

Lemma 2.4. If $L, K, m$ and $q$ are positive integers, then

$$
\frac{1}{L}\left(\frac{1}{K}\langle m, q\rangle \cap \mathbb{Z}\right) \cap \mathbb{Z}=\frac{1}{L K}\langle m, q\rangle \cap \mathbb{Z}
$$

Then, it suffices to find $L_{1}, q_{1}, m$ three pairwise coprime positive integers such that $S=$ $\frac{1}{L_{1}}\left\langle m, q_{1}\right\rangle \cap \mathbb{Z}$, to get a Toms building block if $q_{1}$ is not prime. To show, look at $\left\langle m, q_{1}\right\rangle$. By using the proof of Lemma 2.3 we outlined above, chose $n_{0}$ so that $a_{n_{0}}$ is prime, and greater than both $L_{1}$ and $m q_{1}-m-q_{1}$. Then, define $q_{2}=a_{n_{0}}, L_{2}=n_{0}$. Hence, $q_{2}$ is prime, $q_{2}, m, L_{2}$ are pairwise coprime, $q_{2}$ is coprime to $L_{1}$, and $\left\langle m, q_{1}\right\rangle=\frac{1}{L_{2}}\left\langle m, q_{2}\right\rangle \cap \mathbb{Z}$. Thus, we apply Lemma 2.4 the end the argument. 
In the particular case of an irreducible numerical semigroup $S$, if there exists a Toms decomposition $S=\bigcap_{i=1}^{N}\left(\frac{1}{L}\left\langle q_{i}, m_{i}\right\rangle \cap \mathbb{Z}\right)$, then clearly

$$
S=\frac{1}{L}\langle q, m\rangle \cap \mathbb{Z}
$$

for $L, q, m \in \mathbb{Z}^{+}$, pairwise coprime and $q$ prime.

Notice that having Toms decomposition for irreducible numerical semigroups does not guarantees decomposition for arbitrary numerical semigroups, since it is not clear whether -for any such a decomposition- there exists $L$, coprime to each $q_{1}, \ldots, q_{N}, m_{1}, \ldots, m_{N}$, such that $\bigcap_{i=1}^{N}\left(\frac{1}{L_{i}}\left\langle q_{i}, m_{i}\right\rangle \cap \mathbb{Z}\right)=\frac{1}{L}\left(\bigcap_{i=1}^{N}\left\langle q_{i}, m_{i}\right\rangle\right) \cap \mathbb{Z}$.

\section{NumericAl SEMigroups GENERATED By INTERVALS}

In this section we present the semigroups generated by intervals of nonnegative integers, that is to say, semigroups of the form

$$
S=\langle a, a+1, \ldots, a+x\rangle=\left\{\sum_{i=0}^{x} n_{i}(a+i): n_{i} \in \mathbb{Z}^{+}\right\} \subseteq \mathbb{Z}^{+} .
$$

Note that if $x \geq a$, then $S=\{a, a+1, \ldots\}=a+\mathbb{Z}^{+}=\langle a, a+1, \ldots, 2 a-1\rangle$; thus we may assume that $x \leq a-1$. For such semigroups, García-Sánchez and Rosales [4] computed the Frobenius number and gave a characterization of the numerical semigroups generated by intervals that are symmetric.

Notation 3.1. $\lceil a\rceil$ denote the least integer greater than or equal to $a$.

Next result characterizes which numerical semigroups generated by intervals are irreducible.

Proposition 3.2. Let $S=\langle a, a+1, \ldots, a+t\rangle$ be a numerical semigroup generated by an interval with $a, t$ both integers, $a \geq 3, t \geq 2$. For $t=a-1,\langle 3,4,5\rangle$ is the only irreducible semigroup generated by an interval. If $2 \leq t \leq a-2$, then $S$ is irreducible if and only if $t$ divides $a-2$.

Proof. Let us consider $S=\langle a, a+1, \ldots, a+t\rangle, a \geq 3, t \geq 2$. If $t \geq a, S$ is not written with minimal number of generators. If $t=a-1$, then $S=\mathbb{Z}^{+} \backslash\{1, \ldots, a-1\}$ with $C(S)=a-1$, and the only irreducible case with $t=a-1$ is $\langle 3,4,5\rangle=\mathbb{Z}^{+} \backslash\{1,2\}$ with Frobenius number $C(S)=2$; indeed $\langle 3,4,5\rangle$ is obviously maximal in the set of all numerical semigroups with Frobenius number 2, and according to [8, Theorem 1], $\langle 3,4,5\rangle$ is irreducible. For any other numerical semigroup $S$ such that $t=a-1$, we consider $S^{\prime}=S \cup\{C(S)-1\}=S \cup\{a-2\}$. First, $S^{\prime}$ contains strictly $S$ as $a-2$ does not belong to $S$; secondly $S^{\prime}$ has the same Frobenius number as $S$, namely $C\left(S^{\prime}\right)=C(S)=a-1$, as 1 is not in $S^{\prime}$. Thus $S$ is not maximal in the set of all numerical semigroups with Frobenius number $C(S)$, and consequently $S$ is not irreducible.

In particular we have proved that if $a \geq 4$ and $S=\langle a, a+1, \ldots, a+t\rangle$ is irreducible, then $2 \leq t \leq a-2$. Now we will show the second part of the statement. We start by proving 
that if $S$ is irreducible, then $t$ divides $a-2$. To see this, observe that $S$ has the following structure:

$$
\begin{aligned}
& \langle a, a+1, \ldots, a+t\rangle=\{0,[a, a+1, \ldots, a+t], \ldots,[2 a, 2 a+1, \ldots, 2(a+t)], \ldots \\
& \ldots,[(x-1) a, \ldots,(x-1)(a+t)], \ldots,[C(S)+1, x a, x a+1, \ldots, x(a+t)], \cdots\},
\end{aligned}
$$

where $x$ is an integer greater than or equal to 2 , and the sequences between brackets consists of consecutive numbers. According to the above argument, $C(S)-1$ must be contained in $S$ if $S$ is irreducible. This only occurs when the last gap in $S$ consists only of the integer $C(S)$, i.e. $C(S)-1=(x-1)(a+t)=x a-2$.

The latter yields $x(a+t)-x a=a+t-2$, and further $x=1+\frac{a-2}{t}$. Thus, as $x$ is an integer, $t$ must to divide $a-2$.

Conversely, suppose that $t$ divides $a-2$. By [4, Corollary 5], $C(S)=\left\lceil\frac{a-1}{t}\right\rceil a-1$. Then, as $\frac{a-1}{t}=\frac{a-2}{t}+\frac{1}{t}$, we get $\left\lceil\frac{a-1}{t}\right\rceil a=\left(\frac{a-2}{t}+1\right) a$, which is an even number if $a \geq 3$. Thus, $C(S)$ is odd. By [4, Theorem 6], $S$ symmetric, whence $S$ is irreducible, as desired.

The proof of Proposition 3.2 also establishes the following result.

Corollary 3.3. If $S=\langle a, a+1, \ldots, a+t\rangle$ is irreducible, then $C(S)=(a-1)+a \frac{a-2}{t}$.

Note that this number is odd whatever $a$ and $t$ are such that $t$ divides $a-2$.

Now, we get the main result of this section, showing that irreducible numerical semigroups generated by an interval enjoy Toms decompositions.

Theorem 3.4. Let $S=\langle a, a+1, a+2, \ldots, a+t\rangle$, with $a, t$ positive integers such that $a \geq 4$, $2 \leq t \leq a-2$ and $t$ divides $a-2$. Then $S$ has Toms decomposition. More concretely the following identities hold:

(1) If $a$ is odd then $S=\frac{1}{t}\langle a, a+t\rangle \cap \mathbb{Z}$.

(2) If $a$ is even and $t$ is odd then $S=\frac{1}{t}\langle a, a+t\rangle \cap \mathbb{Z}$.

(3) If both $a$ and $t$ are even then $S=\frac{1}{a(t-1)+1}\langle a, C(S)(t-1)+(a+t)\rangle \cap \mathbb{Z}$.

Proof.

(1) If $a$ is odd, we have g.c.d. $(a, t)=$ g.c.d. $(a, a+t)=$ g.c.d. $(a+t, t)=1$. As

$$
a+i=\frac{(t-i) a+i(a+t)}{t} \text { with } i \in\{0, \ldots, t\},
$$

we have $S \subseteq \frac{1}{t}\langle a, a+t\rangle \cap \mathbb{Z}$.

For the reverse inclusion, by [8, Theorem 1], we will see that $C(S) \notin \frac{1}{t}\langle a, a+t\rangle \cap \mathbb{Z}$. Suppose that $C(S) \in \frac{1}{t}\langle a, a+t\rangle \cap \mathbb{Z}$. If we denote $\eta=C(S)-a$. Then there exists 
$\alpha^{\prime}, \beta^{\prime} \in \mathbb{Z}^{+}$such that

$$
a+\eta=\frac{\alpha^{\prime} a+\beta^{\prime}(a+t)}{t} .
$$

Now, we write $\beta^{\prime}=k a+\beta$, with $k \in \mathbb{Z}^{+}$and $0 \leq \beta \leq a-1$. If we replace $\alpha^{\prime}$ by $\alpha=\alpha^{\prime}+k(a+t)$ and $\beta^{\prime}$ by $\beta$, then

$$
a+\eta=\frac{\alpha a+\beta(a+t)}{t},
$$

where $\alpha, \beta \in \mathbb{Z}^{+}$with $0 \leq \beta \leq a-1$ and $\alpha \geq 0$. Therefore

$$
t(a+\eta)=\alpha a+\beta(a+t),
$$

whence $\eta t \equiv \beta t(\bmod a)$. As g.c.d. $(t, a)=1$, we have $\eta \equiv \beta(\bmod a)$, and so $\eta=\beta+a n$ for some $n \geq 0$. Thus, Corollary 3.3 and the restriction $0 \leq \beta \leq a-1$ imply that $n=\frac{a-2}{t}-1$, and so $\beta=a-1$. Substituting in (3.2) we get

$$
a(a-2)+t(a-1)=\alpha a+(a-1)(a+t),
$$

so that $\alpha=-1$, contradicting the assumption.

(2) If $a$ is even and $t$ is odd, we have g.c.d. $(a, t)=$ g.c.d. $(a, a+t)=$ g.c.d. $(t, a+t)=1$. Suppose that g.c.d. $(a, t)=d$. Then $d$ would divide $t$, but then $d$ would as well divide $a-2$, since $S$ is irreducible, which would result in $d=1$ or $d=2$. However $d=2$ is a contradiction, since $t$ is odd. Thus, g.c.d. $(a, t)=1$. Now the proof of case (1) give us the desired result.

(3) If both $a$ and $t$ are even, we will prove that

$$
S=\frac{1}{a(t-1)+1}\langle a, C(S)(t-1)+a+t\rangle \cap \mathbb{Z} .
$$

We denote $L=a(t-1)+1$ and $q=C(S)(t-1)+a+t$. We have that g.c.d. $(L, a)=1$. Notice that by Corollary 3.3

$$
q=\left(a-1+a \frac{a-2}{t}\right)(t-1)+a+t=a\left((a-2) \frac{t-1}{t}+t\right)+1 .
$$

Thus, g.c.d. $(a, q)=1$.

As $t\left(q-L\left(\frac{a-2}{t}+1\right)\right)=a(t-1)+2$, we have that $t q=L(a-1+t)+1$, and therefore g.c.d $(L, q)=1$.

Now, we will prove that

$$
S=\langle a, a+1, \ldots, a+t\rangle=\frac{1}{a(t-1)+1}\langle a, C(S)(t-1)+(a+t)\rangle \cap \mathbb{Z}
$$

holds. First, we will show that the identity

$$
a+\eta=\frac{\alpha_{\eta} a+\beta_{\eta}(C(S)(t-1)+a+t)}{a(t-1)+1}
$$

holds for $\eta=0,1, \ldots, t$ and a related couple of nonnegative integers $\alpha_{\eta}, \beta_{\eta}$. Notice that, if we define $\beta_{\eta}=\eta$, then (3.3) is equivalent to

$$
\alpha_{\eta}=\frac{(a(t-1)+1)(a+\eta)-\eta\left(\left(a-1+a \frac{a-2}{t}\right)(t-1)+a+t\right)}{a}=
$$




$$
=\eta\left(\frac{a-2}{t}+1\right)+a(t-\eta-1)+1 .
$$

For $\eta=0,1, \ldots, t-1, \alpha_{\eta}$ is obviously a nonnegative integer; and $\eta=t$ yields $\alpha_{t}=$ $a-2+t-a+1=t-1>0$. Thus,

$$
\langle a, a+1, \ldots, a+t\rangle \subseteq \frac{1}{a(t-1)+1}\langle a, C(S)(t-1)+(a+t)\rangle \cap \mathbb{Z} .
$$

Now, by $[8$, Theorem 1$]$, we only need to check that

$$
C(S) \notin \frac{1}{a(t-1)+1}\langle a, C(S)(t-1)+(a+t)\rangle \cap \mathbb{Z} .
$$

So, we will see that (3.3) fails for $\eta=a \frac{a-2}{t}-1$, whenever $\alpha_{\eta}, \beta_{\eta}$ are both nonnegative integers. Writing (3.3) as

$$
(a(t-1)+1)(a+\eta)=\alpha_{\eta} a+\beta_{\eta}\left(\left(a-1+a \frac{a-2}{t}\right)(t-1)+a+t\right),
$$

and reducing this identity modulo $a$, one has that $\eta \equiv \beta_{\eta}(\bmod a)$. An analog argument to that of case (1), using Corollary 3.3 and the condition $0 \leq \beta_{\eta} \leq a-1$, ensure that $\beta_{\eta}=a-1$. Substituting the value of $\beta_{\eta}$ in (3.4) and multiplying by $t$, we get first $(a(t-1)+1)(t a+a(a-2)-t)=t \alpha_{\eta} a+(a-1)\left((t a-t+a(a-2))(t-1)+a t+t^{2}\right)$,

that give us

$$
t a+a(a-2)-t=t \alpha_{\eta} a-(t a-t+a(a-2))(t-1)+(a-1)\left(a t+t^{2}\right),
$$

and thus

$$
t a+a^{2}-2 a-t=t \alpha_{\eta} a+2 a t-t+a^{2}-2 a,
$$

whence

$$
t a=t \alpha_{\eta} a+2 a t
$$

Hence,

$$
\alpha_{\eta}=\frac{t a-2 t a}{a t}=-\frac{a t}{a t}=-1
$$

contradicting the assumption. Thus, the result holds.

Remark 3.5. It can be check easily that $S=\langle 3,4,5\rangle=\frac{1}{2}\langle 3,5\rangle \cap \mathbb{Z}$.

Examples 3.6. We have

(1) $S=\langle 6,7,8\rangle=\frac{1}{7}\langle 6,25\rangle \cap \mathbb{Z}$.

(2) $T=\langle 14,15,16,17\rangle=\frac{1}{29}\langle 14,239\rangle \cap \mathbb{Z}$.

(3) $V=\langle 11,12,13,14\rangle=\frac{1}{3}\langle 11,14\rangle \cap \mathbb{Z}$.

(4) $W=\langle 4,5,6\rangle=\frac{1}{7}\langle 4,19\rangle \cap \mathbb{Z}$. 
Remark 3.7. The semigroups (2) and (3) in Examples 3.6 have $q$ prime, whereas $\langle 6,25\rangle$ needs to be transformed using [10, Lemma 3.3.1]. A prime number $q_{S}$ is required, such that $q_{S}=-6+25 L_{S}$, where $q_{S} \geq C(\langle 6,25\rangle)=119, L_{S}$ is coprime to 6 , and both $q$ and $L_{S}$ are coprime to 7 . The least value of $q_{S}$ fulfilling these conditions is $q_{S}=269$, with a related $L_{S}=11$. Thus, by Lemmas 2.3 and 2.4,

$$
S=\langle 6,7,8\rangle=\frac{1}{7}\left(\frac{1}{11}\langle 6,269\rangle\right) \cap \mathbb{Z}=\frac{1}{77}\langle 6,269\rangle \cap \mathbb{Z} .
$$

\section{Other examples having Toms Decomposition}

In this section we will show that the Toms decomposition holds for some kinds of irreducible numerical semigroup with multiplicity 3 and 4, and these appearing as expansions of numerical semigroups generated by an interval.

We begin characterizing when an expansion of a numerical semigroup generated by an interval is irreducible.

Proposition 4.1. Let $a$ odd, $a \geq 5$ and $x>0$. Then $S=\langle a, x+a, 2 x+a, \ldots,(a-2) x+a\rangle$ is an irreducible numerical semigroup if and only if g.c.d. $(a, x)=1$.

Proof. Let $S=\langle a, x+a, 2 x+a, \ldots,(a-2) x+a\rangle$. Then, g.c.d. $(a, x)=1$ is a necessary condition for $S$ to be written with a minimal number of generators. To see this, assume per absurdum that $a=p q$ and $x=k q$ for some positive integers $k, p$ and $q$, where $k \geq 2$ and $p$, $q \geq 3$. Then

$$
S=\langle p q,(k+p) q,(2 k+p) q, \ldots,((p q-2) k+p) q\rangle .
$$

As $1<p<p q-2$, then the sequence $p, k+p, 2 k+p, \ldots,(p q-2) k+p$ contains $p k+p$ and hence $a=p q$ divides the generator $p(k+1) q$, which contradicts $\mu(S)=a-1$.

To prove the converse, we assume that g.c.d. $(a, x)=1$ and first show that the Frobenius number of $S$ is $C(S)=(a-1) x+a$. To see this, let us show that $(a-1) x+a$ does not belong to $S$, but every $n \in S$ such that $n>(a-1) x+a$ is in $S$. Assume at first that $(a-1) x+a$ is in $S$. Then there exist $\alpha_{0}, \ldots, \alpha_{a-2}$, all nonnegative integers, such that

$$
(a-1) x+a=a \alpha_{0}+(x+a) \alpha_{1}+\ldots+((a-2) x+a) \alpha_{a-2} .
$$

That is

$$
(a-1) x+a=x\left(\alpha_{1}+2 \alpha_{2}+\ldots+(a-2) \alpha_{a-2}\right)+a \sum_{i=0}^{a-2} \alpha_{i} .
$$

Assume that $\sum_{i=0}^{a-2} \alpha_{i}=k>1$. Then $x\left(a-1-\left(\alpha_{1}+2 \alpha_{2}+\ldots+(a-2) \alpha_{a-2}\right)\right)=(k-1) a$, but g.c.d. $(a, x)=1$ ensures that $a$ divides $a-1-\left(\alpha_{1}+2 \alpha_{2}+\ldots+(a-2) \alpha_{a-2}\right)$ and consequently $\alpha_{1}+2 \alpha_{2}+\ldots+(a-2) \alpha_{a-2} \leq-1$, which is absurd. Hence, as $\sum_{i=0}^{a-2} \alpha_{i} \leq 0$ is absurd as well, $\sum_{i=0}^{a-2} \alpha_{i}=1$, which means that exactly one of the $\alpha_{i}$ is 1 and every other 0 . But then (4.1) does not hold. Thus $(a-1) x+a \notin S$.

Let us now consider $n=(a-1) x+t, t \geq a+1$. If $t>2 a$, then $n$ can be expressed as $(a-1) x+r+a k$, with $r \in\{a+1, a+2, \ldots, 2 a\}$ and $k$ a positive integer. And $t=2 a$ yields 
$(a-1) x+2 a=2\left(\frac{a-1}{2} x+a\right) \in S$, as $a$ is odd and $2 \leq \frac{a-1}{2}<a-2$. Hence it suffices to show that $(a-1) x+t$ belongs to $S$ for $t \in\{a+1, \ldots, 2 a-1\}$.

Look at the generators of $S$. As $x$ is coprime with $a$, each of the $a-1$ generators belongs to different congruence classes modulo $a$, and the missing class is $-x$ (the one corresponding to $(a-1) x+a$, the Frobenius number of $S)$. Now let us consider $(a-1) x+t \equiv t-x(\bmod a)$. For $t \in\{a+1, \ldots, 2 a-1\}, t-x$ runs through each congruence class modulo $a$, except precisely class $-x$. Hence $(a-1) x+t$ belongs to the same congruence class modulo $a$ as one of the generators, and is greater than this generator. Denote by $\psi$ the suitable generator. Then $(a-1) x+t=\psi+a \xi$, for some $\xi \in \mathbb{Z}^{+}$. Thus $C(S)=(a-1) x+a$.

Let us now show that $S$ is irreducible. Observe that $C(S)$ is odd. We will prove $w(i)+$ $w(a-i+1)=w(a)$ for $i \in\{1, \ldots, a\}$, and by [8, Proposition 3], $S$ is an irreducible numerical semigroup.

We begin proving that

$$
A p(S, a)=\{0, x+a, 2 x+a, \ldots,(a-2) x+a,(a-1) x+2 a\} .
$$

To see this, observe that $w(1)=0$ as $a$ is the least element in $S$, and $w(a)=C(S)+a$, according to a result given by [6, Proposition 10.4]. By definition of $A p(S, a)$ (see Section $2)$, we have to check that $w(i)-a \notin S$ for $i=2, \ldots, a-1$. As $w(i)=(i-1) x+a$, then $w(i)-a=(i-1) x$. Let $j=i-1$ and assume that $j x \in S$ for $j=1,2, \ldots, a-2$. Then there exists $\beta_{0}, \ldots, \beta_{j-1}$, all nonnegative integers, such that $j x=a \beta_{0}+(x+a) \beta_{1}+\ldots+((j-$ 1) $x+a) \beta_{j-1}$ (we exclude $j x+a, \ldots,(a-2) x+a$, as they are greater than $j x$ ) and further $\left(j-\sum_{k=1}^{j-1} \beta_{k} k\right) x=a \sum_{k=0}^{j-1} \beta_{k}$. As g.c.d. $(a, x)=1, a$ divides $j-\sum_{k=1}^{j-1} \beta_{k} k$, which is impossible, since

$$
j-\sum_{k=1}^{j-1} \beta_{k} k<j \leq a-2 .
$$

Thus

$$
A p(S, a)=\{0=w(1), x+a=w(2), \ldots,(a-2) x+a=w(a-1),(a-1) x+2 a=w(a)\}
$$

and, as $w(i)-a=(i-1) x$ and $w(a)=(a-1) x+2 a$, the identity $w(i)+w(a-i+1)=w(a)$ holds, so that $S$ is irreducible.

Finally, we observe that $S$ is written with a minimal number of generators. Assume that some of them -say $l x+a$ for some $l \in\{1, \ldots, a-2\}$ - is a positive integer combination of the remaining generators. Provided that $a$ is the smallest element in $S$, it is a necessary generator, and only those smaller than $l x+a$ take part in this combination. Then, there exists $\gamma_{0}, \ldots$, $\gamma_{l-1}$, nonnegative integers, such that $l x+a=a \gamma_{0}+(x+a) \gamma_{1}+\ldots+((l-1) x+a) \gamma_{l-1}$, and further $\left(l-\sum_{k=1}^{l-1} \gamma_{k} k\right) x=a\left(\sum_{k=0}^{l-1} \gamma_{k}-1\right)$. As g.c.d. $(a, x)=1$, we get the same contradiction as $(4.2)$.

The next result shows that the irreducible numerical semigroups with multiplicity 3 , as well as those appearing as expansions of a numerical semigroup generated by an interval have Toms decomposition.

\section{Theorem 4.2.}


(1) If $x$ is a strictly positive integer and $x$ not a multiple of 3 , then $S=\langle 3, x+3,2 x+3\rangle$ has Toms decomposition

$$
S=\frac{1}{x+2}\langle x+3,2 x+3\rangle \cap \mathbb{Z} .
$$

(2) Let $S=\langle a, x+a, 2 x+a, \ldots,(a-2) x+a\rangle$, a numerical semigroup where g.c.d. $(a, x)=1$, $a$ is odd, $a \geq 5$ and $x>0$. Then $S$ has Toms decomposition

$$
S=\frac{1}{a-2}\langle a,(a-2) x+a\rangle \cap \mathbb{Z} .
$$

Proof.

(1) By [8, Theorem 7], the only irreducible numerical semigroup with $\mu(S)=m(S)=3$ is $S=\langle 3, x+3,2 x+3\rangle$, where $x$ is a strictly positive integer such that $x$ is not multiple of 3. Let $k=3, m=x+3$ and $q=2 x+3$ and let us implement $L=\frac{q+m}{k}=$ $\frac{(2 x+3)+(x+3)}{3}=x+2$. Then, $m$ and $L$ are obviously coprime. Also, $L$ and $q$ are coprime, as any non trivial common factor would divide $1=2 L-q$. Assume that $p$ divides both $m$ and $q$ for some $p$ prime; then $p$ divides $3=2 m-q$, which entails $p=3$ and contradicts $3 \nmid x$. Thus g.c.d. $(m, q)=1$. We will prove now that

$$
\frac{1}{x+2}\langle x+3,2 x+3\rangle \cap \mathbb{Z}=\langle 3, x+3,2 x+3\rangle .
$$

As,

and

$$
\begin{gathered}
3=\frac{(2 x+3)+(x+3)}{x+2}, \\
x+3=\frac{1}{x+2}((x+2)(x+3)+0 \cdot(2 x+3))
\end{gathered}
$$

$$
2 x+3=\frac{1}{x+2}(0 \cdot(x+3)+(x+2)(2 x+3)),
$$

we have $\frac{1}{x+2}\langle x+3,2 x+3\rangle \cap \mathbb{Z} \supseteq\langle 3, x+3,2 x+3\rangle$.

To see the converse, assume that $\frac{a(x+3)+b(2 x+3)}{x+2}$ is an integer for $a, b$ both nonnegative integers; assume furthermore that $a \geq b$. Then

$$
\frac{a(x+3)+b(2 x+3)}{x+2}=b \frac{(x+3)+(2 x+3)}{x+2}+\frac{(a-b)(x+3)}{x+2}=3 b+\frac{(a-b)(x+3)}{x+2} .
$$

As g.c.d. $(x+2, x+3)=1$, there exists $d \in \mathbb{Z}^{+}$such that $d=\frac{a-b}{x+2}$ and consequently $\frac{a(x+3)+b(2 x+3)}{x+2}=3 b+d(x+3)$.

A similar argument with $b \geq a$ shows that, for some $g \in \mathbb{Z}^{+}$,

$$
\frac{a(x+3)+b(2 x+3)}{x+2}=3 a+g(2 x+3) \text {. }
$$


Thus, $\frac{1}{x+2}\langle x+3,2 x+3\rangle \cap \mathbb{Z} \subseteq\langle 3, x+3,2 x+3\rangle$ and reciprocal inclusion yields

$$
\frac{1}{x+2}\langle x+3,2 x+3\rangle \cap \mathbb{Z}=\langle 3, x+3,2 x+3\rangle,
$$

completing the proof.

(2) Let us denote by $T$ the numerical semigroup $\frac{1}{a-2}\langle a,(a-2) x+a\rangle \cap \mathbb{Z}$. As $a$ is odd, $a-2, a$ and $(a-2) x+a$ are pairwise coprime and both $a$ and $(a-2) x+a$ belong to $T$. To check that the remaining generators of $S$ are in $T$, it is enough to find two suitable nonnegative integers $\varphi, \chi$ such that the equation $k x+a=\frac{1}{a-2}(\varphi a+\chi((a-2) x+a))$ holds for each $k \in\{1, \ldots, a-3\}$. Choose $\chi=k$, whence the identity reduces to $a-2=\varphi+k$. As $k$ runs increasingly from 1 to $a-3$, there exists a related $\varphi$ running decreasingly from $a-3$ to 1 . Thus $T \supseteq S$.

The converse is easy to prove, checking that $C(S) \notin T$. Assume per absurdum that $C(S) \in T$. Then there exist $\varkappa, \varrho$ both non-negative integers such that

$$
(a-1) x+a=\frac{\varkappa a+\varrho((a-2) x+a)}{a-2} .
$$

We deduce that $\varrho<a-1$. As $(4.3)$ is $(a-2)((a-1)-\varrho) x+((a-2)-\varrho) a=\varkappa a$, $a$ must divide $(a-2)((a-1)-\varrho) x$, which is impossible as g.c.d. $(a, x)=1$ and $a>$ $a-1-\varrho$ for $\varrho<a-1$. Thus $C(S) \notin T$ and $S=T$.

As a consequence, we obtain an expression of Toms decomposition for other families of numerical semigroups, as follows.

Corollary 4.3. If $x$ is a strictly positive integer then $S=\langle 3,3 x+2,6 x+1\rangle$ has Toms decomposition $S=\frac{1}{3 x+1}\langle 3 x+2,6 x+1\rangle \cap \mathbb{Z}$.

Proof. By assumption, $3 x-1$ is a strictly positive integer, and it is not multiple of 3 . Then, the result holds by part (1) of Theorem 4.2.

Example 4.4. We have

(1) $S=\langle 3,5,7\rangle=\frac{1}{4}\langle 5,7\rangle \cap \mathbb{Z}$.

(2) If $S=\langle 5, x+5,2 x+5,3 x+5\rangle$ with g.c.d. $(5, x)=1$ and $x>0$, then $S=\frac{1}{3}\langle 5,3 x+5\rangle \cap \mathbb{Z}$.

Notice that no such identity has been found for $S=\langle a, x+a, 2 x+a, \ldots,(a-2) x+a\rangle$, where g.c.d. $(a, x)=1$ and $a$ is even.

Next result shows that the irreducible numerical semigroups with multiplicity 4 have Toms decomposition.

Proposition 4.5. Let $S=\langle 4, x+2, x+4\rangle$, where $x$ is an odd integer greater than or equal to 3 . Then $S=\frac{2}{(x+3)}\langle x+2, x+4\rangle \cap \mathbb{Z}$. 
Proof. By [8, Theorem 9], $S$ is an irreducible numerical semigroup, and $m(S)=4$. Now, the proof is analog to that of Theorem 4.2(1), by fixing $k=4, m=x+2, q=x+4$ and

$$
L=\frac{q+m}{k}=\frac{(x+4)+(x+2)}{4}=\frac{x+3}{2} \in \mathbb{Z}^{+} .
$$

Example 4.6. We have $\langle 4,7,9\rangle=\frac{1}{4}\langle 7,9\rangle \cap \mathbb{Z}$.

\section{ACKNOWLEDGMENTS}

Part of this work was done during a visit of the second author to the Departamento de Matemáticas de la Universidad de Cádiz (Spain), through a grant of the Erasmus-Socrates Program of the European Community. His contribution is contained in his Master Degree Thesis, done under the supervision of E. Pardo in Fall 2004. The second author thanks the host center for its warm hospitality.

\section{REFERENCES}

[1] V. Barucci, D.E. Dobbs and M. Fontana, Maximality properties in numerical semigroups and applications to one-dimensional analytically irreducible local domains, Memoirs Amer. Math. Soc. 598 (1997).

[2] G. Elliott and J. Villadsen, Perforated ordered $K_{0}$-groups, Canad. J. Math. 52(6)(2000), 11641191.

[3] R. Fröberg, G. Gottlieb and R. HÄGgkvist, On numerical semigroups, Semigroup Forum 35 (1987), 63-83.

[4] P.A. García-SÁnchez and J.C. Rosales, Numerical semigroups generated by intervals, Pacific J. Math. 191(1) (1999), 75-83.

[5] M.A. Moreno, J. Nicola, E. Pardo, H. Thomas, A negative answer to a question of Toms, Preprint $(2005)$.

[6] J.C. Rosales and P.A. García-SÁnchez, "Finitely generated commutative monoids", Nova Science Publishers New York (1999).

[7] J.C. Rosales And M.B. Branco, Decomposition of a numerical semigroup as an intersection of irreducible numerical semigroups, Bull. Belg. Math. Soc. Simon Stevin 9(3) (2002), 373-381.

[8] J.C. Rosales, M.B. Branco, Irreducible numerical semigroups, Pacific J. Math. 209(1) (2003), 131143.

[9] M. RøRdam, J. Villadsen, On the ordered $K_{0}$-group of universal free product $C^{*}$-algebras, $K$-Theory 15 (1998), 307-322.

[10] A.Toms, Ph.D.Thesis, University of Toronto (2001).

[11] A. Toms, Strongly perforated $K_{0}$-groups of simple $C^{*}$-algebras, Canad. Math. Bull. 46(3) (2003), 457472.

[12] J.Villadsen, Simple $C^{*}$-algebras with perforation, J. Funct. Anal. 154(1)(1998), 110-116. 
Departamento de Matemáticas, Universidad de Cádiz, Apartado 40, 11510 Puerto Real (CÁdiz), SPAin.

E-mail address: mariangeles.moreno@uca.es

Département de Mathématiques, Université de Génève, $2-4$ Rue du Lièvre, C.P. 64, 1211 GÉNÈve 4, Switzerland.

Current address: Departamento de Matemáticas, Universidad de Cádiz, Apartado 40, 11510 Puerto Real (Cádiz), Spain.

E-mail address: jacnicola@hotmail.com

Departamento de Matemáticas, Universidad de Cádiz, Apartado 40, 11510 Puerto Real (CÁDIZ), SPAIN.

E-mail address: enrique.pardo@uca.es

URL: http://www.uca.es/dept/matematicas/PPersonales/PardoEspino/EMAIN. HTML 